\title{
Unveiling the impurity band induced ferromagnetism in the magnetic semiconductor (Ga,Mn)As
}

\author{
Masaki Kobayashi,,${ }^{1,2, *}$ Iriya Muneta, ${ }^{3}$ Yukiharu Takeda, ${ }^{4}$ Yoshihisa Harada, ${ }^{5}$ Atsushi Fujimori, ${ }^{6}$ Juraj Krempaský, ${ }^{1}$ \\ Thorsten Schmitt, ${ }^{1}$ Shinobu Ohya, ${ }^{3}$ Masaaki Tanaka, ${ }^{3}$ Masaharu Oshima,${ }^{2}$ and Vladimir N. Strocov ${ }^{1}$ \\ ${ }^{1}$ Swiss Light Source, Paul Scherrer Institut, CH-5232 Villigen PSI, Switzerland \\ ${ }^{2}$ Department of Applied Chemistry, School of Engineering, University of Tokyo, 7-3-1 Hongo, Bunkyo-ku, Tokyo 113-8656, Japan \\ ${ }^{3}$ Department of Electrical Engineering and Information Systems, University of Tokyo, 7-3-1 Hongo, Bunkyo-ku, Tokyo 113-8656, Japan \\ ${ }^{4}$ Synchrotron Radiation Research Unit, Japan Atomic Energy Agency, Sayo-gun, Hyogo 679-5148, Japan \\ ${ }^{5}$ Institute for Solid State Physics, University of Tokyo, Kashiwanoha, Kashiwa, Chiba 277-8561, Japan \\ ${ }^{6}$ Department of Physics, University of Tokyo, 7-3-1 Hongo, Bunkyo-ku, Tokyo 113-0033, Japan
}

(Received 24 January 2013; published 19 May 2014)

\begin{abstract}
(Ga,Mn)As is a paradigm of a diluted magnetic semiconductor which shows ferromagnetism induced by doped hole carriers. With a few controversial models emerging from numerous experimental and theoretical studies, the mechanism of the ferromagnetism in $(\mathrm{Ga}, \mathrm{Mn})$ As still remains a puzzling enigma. In this article, we use soft x-ray angle-resolved photoemission spectroscopy to positively identify the ferromagnetic Mn $3 d$-derived impurity band (IB) in (Ga,Mn)As. The band appears dispersionless and hybridized with the light-hole band of the host GaAs. These findings conclude the picture of the valence-band structure of (Ga,Mn)As disputed for more than a decade. The nondispersive character of the IB and its location in vicinity of the valence-band maximum indicate that the Mn $3 d$-derived IB is formed as a split-off Mn-impurity state predicted by the Anderson impurity model. Responsible for the ferromagnetism is predominantly the transport of hole carriers in the IB.
\end{abstract}

DOI: 10.1103/PhysRevB.89.205204

PACS number(s): 75.50.Pp, 71.55.Eq, 79.60.-i, 85.75.-d

\section{INTRODUCTION}

Ferromagnetic diluted magnetic semiconductors (DMSs) are formed by substitution of several percent of cation sites in a host semiconductor by magnetic impurities. Because the carriers in DMSs are considered to mediate the magnetic interaction between the magnetic ions [1], these materials are of key importance for "spintronics" aiming at development of advanced functional devices to control the spin degree of freedom of the carriers. Due to the mediation mechanism, the ferromagnetism (FM) in DMS is called carrier-induced FM. This mechanism is equipped with the capability of manipulating both the electron charge and spin degrees of freedom in functional spintronic devices. The III-V based DMS $(\mathrm{Ga}, \mathrm{Mn}) \mathrm{As}$ is a prototype ferromagnetic DMS, which has been intensively studied from both fundamental and applicational points of view [2]. Although the Curie temperature $\left(T_{\mathrm{C}}\right)$ of $(\mathrm{Ga}, \mathrm{Mn}) \mathrm{As}$ is at present lower than room temperature, applications of $(\mathrm{Ga}, \mathrm{Mn})$ As to functional spintronic devices have been established.

Understanding the mechanism of the carrier-induced FM is of paramount importance for further development of spintronic device applications with DMS. Several theoretical models for carrier-induced FM in (Ga,Mn)As have been proposed [1]. In the limit where the hole carriers are considered nearly free or itinerant, the Zener $p-d$ exchange model has been suggested based on a mean-field theory point of view [3,4]. Here, the Mn $3 d$ acceptor level is located above the Fermi level $\left(E_{\mathrm{F}}\right)$ and merges into the valence band (VB) of the host GaAs through its hybridization, leading to the exchange-split VB of host GaAs crossing $E_{\mathrm{F}}$. This model can explain the carrier-concentration

\footnotetext{
*Present address: Photon Factory, Institute of Materials Structure Science, High Energy Accelerator Research Organization, Tsukuba, Ibaraki 305-0801, Japan; masakik@ post.kek.jp
}

dependence of $T_{\mathrm{C}}$ measured on an electric-field transistor structure using $(\mathrm{Ga}, \mathrm{Mn}) \mathrm{As}$ [5]. In the opposite limit where the hole carriers are strongly localized around the magnetic impurity, the Mn $3 d$ impurity band (IB) model has been proposed [6-9]. Here, in contrast to the Zener model, the IB intersects $E_{\mathrm{F}}$. This model can explain the experimental results of vacuum-ultraviolet angle-resolved photoemission spectroscopy (ARPES) [10], hard x-ray ARPES [11], and optical spectroscopy suggesting that $E_{\mathrm{F}}$ resides in the IB [12], as well as the results of resonant tunneling spectroscopy indicating that $E_{\mathrm{F}}$ is located above the valence-band maximum (VBM) of the host GaAs [13,14]. Additionally, as a model bridging from the localized hole carriers to the delocalized hole carriers causing the FM, the percolation theory of bound magnetic polarons (BMPs) has been proposed $[15,16]$. The model well explains the temperature dependence of the transport property of $(\mathrm{Ga}, \mathrm{Mn})$ As with low carrier concentration [16], and is likely relevant for an understanding of the nanoscale phase separation [5] and inhomogeneous growth of magnetic domains near $T_{\mathrm{C}}$ [17]. Here, to address the issue of the VB structure involving Mn $3 d \mathrm{IB}$, we report the results of soft $\mathrm{X}$-ray (SX) ARPES measurements of $(\mathrm{Ga}, \mathrm{Mn})$ As that take advantage of the enhanced probing depth combined with the elemental and chemical state specificity achieved with this technique [18,19]. Our $\mathrm{Ga}_{1-x} \mathrm{Mn}_{x}$ As samples, with a low Mn concentration just above the onset of FM [8,9], have an advantage in that they provide an authentic picture of FM in $\mathrm{Ga}_{1-x} \mathrm{Mn}_{x}$ As without any ambiguities due to potential formation of MnAs clusters at large Mn concentrations.

\section{EXPERIMENTAL}

$\mathrm{Ga}_{1-x} \mathrm{Mn}_{x}$ As $(x=0.025)$ thin films with a thickness of $100 \mathrm{~nm}$ were grown on a $\operatorname{GaAs}(001)$ substrate at $275^{\circ} \mathrm{C}$ under ultrahigh vacuum by the molecular beam epitaxy method. To avoid surface oxidation, after the deposition of a 
$\mathrm{Ga}_{1-x} \mathrm{Mn}_{x}$ As layer the sample was covered by an amorphous As capping layer with a thickness of 5-10 $\AA$ to produce the structure As $/ \mathrm{Ga}_{0.975} \mathrm{Mn}_{0.025} \mathrm{As} / \mathrm{GaAs}$ (buffer)/GaAs(001). The Curie temperature $T_{\mathrm{C}}$ of our sample was $\sim 35 \mathrm{~K}$, as determined by the Arrott plot of the magnetic circular dichroism. The SX-ARPES experiments were conducted at the ADRESS beamline [20] of the Swiss Light Source using the SX-ARPES end station [21]. The measurements were carried through the As capping layer without its thermal desorption, thereby accessing the native $(\mathrm{Ga}, \mathrm{Mn})$ As electronic structure without potential formation of MnAs clusters. In the photon energy $(h v)$ range from 350 to $1000 \mathrm{eV}$, the combined beamline and analyzer energy resolution varied from 50 to $150 \mathrm{meV}$. With our experimental geometry [21], the analyzer slit was oriented to lie in the measurement plane. The measurements used linear vertical $(p)$ and horizontal $(s)$ polarizations of the incident beam. The measurements were performed under ultrahigh vacuum of $5.0 \times 10^{-11} \mathrm{mbar}$ at a temperature of $11 \mathrm{~K}$, which essentially eliminated the effects of thermal broadening as well as electron-phonon scattering destroying the coherent spectral weight [21]. The $\mathrm{Mn} L_{2,3}$ x-ray absorption spectroscopy (XAS) spectra were measured in the total-electron-yield mode.

\section{RESULTS AND DISCUSSION}

\section{A. Band dispersion around the $\Gamma$ point}

Our $\mathrm{Ga}_{1-x} \mathrm{Mn}_{x}$ As thin-film sample was capped by an amorphous As layer, which well protects a GaAs underlayer against oxidization, exposure to deionized water, and annealing up to $180^{\circ} \mathrm{C}$ [22]. In this report, we demonstrate that the use of SX-ARPES with photon energies $h v$ towards $1 \mathrm{keV}$ allows the observation of the bulk band dispersion of the $\mathrm{Ga}_{1-x} \mathrm{Mn}_{x} \mathrm{As}$ underlayer through the amorphous passivation layer, with any contributions from their interface being hardly seen in the spectra (see also Figs. S1 and S2, and the movie in the Supplemental Material [23]). Details of the SX-ARPES experiment illustrating its penetrating ability through capping As layers have been published elsewhere [24]. These results exclude any possible surface As capping layer or $(\mathrm{Ga}, \mathrm{Mn}) \mathrm{As} / \mathrm{As}$ interface contributions in our ARPES spectra. Hereafter, we shall focus on the bulk electronic structure of $(\mathrm{Ga}, \mathrm{Mn}) \mathrm{As}$ seen in our SX-ARPES experiments.

Figure 1 shows the binding energy $\left(E_{B}\right)$ vs momentum $(k)$ plots along the $\Gamma-\mathrm{K}-\mathrm{X}$ symmetry line of the Brillouin zone (BZ) measured with $p$ and $s$ polarizations at $h v$ of $890 \mathrm{eV}$. Due to the wave-function symmetry properties, the light-hole (LH) and split-off (SO) bands of the host GaAs show up with the $p$ polarization [Fig. 1(a)], whereas the heavy-hole $(\mathrm{HH})$ band pops up in the image taken with the $s$ polarization [Fig. 1(b)]. The LH and HH bands become almost degenerated along the $\Gamma-X-\Gamma$ line, as shown in Fig. 1(c). These dispersive bands form the band manifold characteristic of the GaAs band structure. Our results are consistent with the previous ARPES experiments and band calculations on GaAs [25-27]. Figures 1 (d) and 1(e) display the energy distribution curves (EDCs) along the $\Gamma$-K-X symmetry line around the $\Gamma$ point with $p$ and $s$ polarizations, respectively. Note that although the LH and $\mathrm{HH}$ bands approach $E_{\mathrm{F}}$ in the vicinity of the $\Gamma$ point, they do
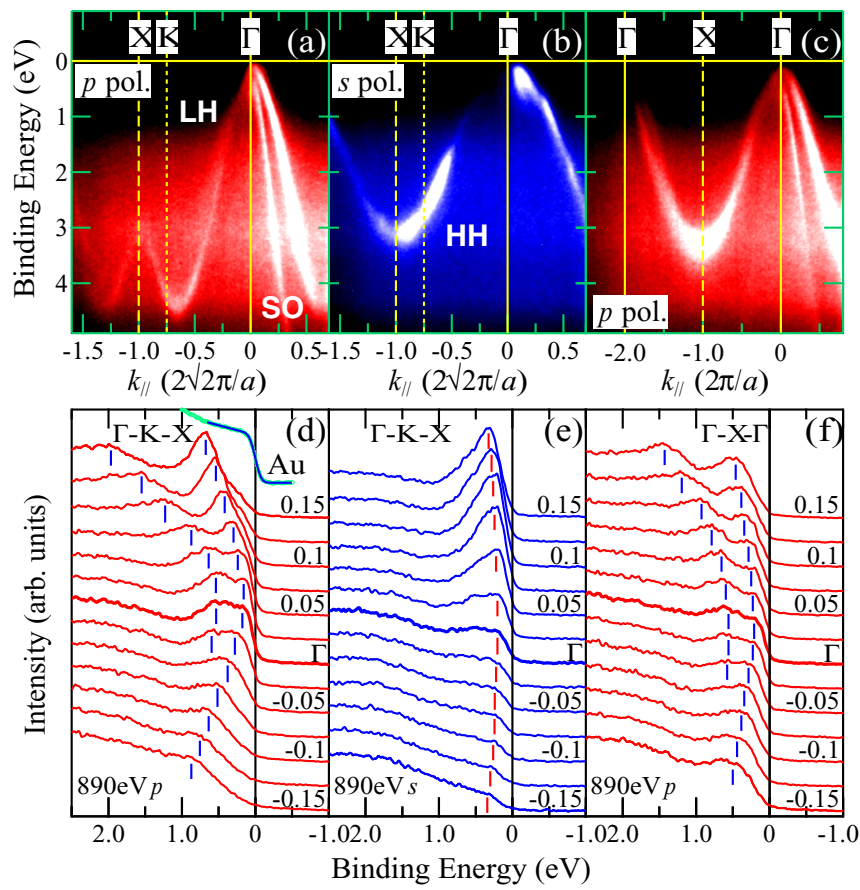

FIG. 1. (Color online) Band dispersion near the $\Gamma$ point in the $\mathrm{Ga}_{0.975} \mathrm{Mn}_{0.025} \mathrm{As}$ thin film. The incident photon energy is $h v=$ $890 \mathrm{eV}$. (a, b) ARPES images along the $\Gamma-\mathrm{K}-\mathrm{X}$ line measured with $p$ and $s$ polarizations, respectively. The light-hole (LH) and split-off (SO) bands are clearly seen with the $p$ polarization, while the heavy-hole $(\mathrm{HH})$ band is active with the $s$ polarization. (c) ARPES image along the $\Gamma-\mathrm{X}-\Gamma$ line. (d)-(f) Energy-distribution curves (EDCs) around the $\Gamma$ point corresponding to (a) $-(\mathrm{c})$. The vertical bars are guides for the eyes tracing the spectra structures. The Fermi-level position has been measured with Au foil in electrical contact with the sample. These results identify the textbook manifold of $\mathrm{LH}, \mathrm{HH}$, and SO bands of GaAs.

not intersect $E_{\mathrm{F}}$ even at their top. The same is observed along the $\Gamma-\mathrm{X}-\Gamma$ line, as shown in Fig. 1(c). These results provide unambiguous evidence that the VBM of host GaAs is located below $E_{\mathrm{F}}$, which is consistent with the results of resonant tunneling spectroscopy [13] and immediately dismisses the Zener $p$ - $d$ exchange model $[3,4]$.

Furthermore, we note a small but finite spectral weight at $E_{\mathrm{F}}$, which is a direct observation of finite density of states at $E_{\mathrm{F}}$ in $(\mathrm{Ga}, \mathrm{Mn})$ As. Figure 2 shows a comparison of the EDCs in the vicinity of the $\Gamma$ point between $(\mathrm{Ga}, \mathrm{Mn}) \mathrm{As}$ and pure GaAs. The slope near $E_{\mathrm{F}}$ in $(\mathrm{Ga}, \mathrm{Mn}) \mathrm{As}$ is smaller than that in GaAs, manifesting a finite intensity near $E_{\mathrm{F}}$ in (Ga,Mn)As. This result identifies an additional spectral weight at $E_{\mathrm{F}}$ due to the Mn doping in $(\mathrm{Ga}, \mathrm{Mn}) \mathrm{As}$. This fact, consistent with the metallic conductivity of $(\mathrm{Ga}, \mathrm{Mn}) \mathrm{As}$, has been noted in the previous hard $\mathrm{x}$-ray ARPES study with its large probing depth [11] but was missed by all previous low-energy photoemission experiments, including in situ ones [10,11,28,29]. The reason is probably the surface effects: The conventional photoemission experiments on semiconductor surfaces require their special treatment (such as ion sputtering and annealing) to remove the adsorbed surface contamination molecules. As these surface treatments are basically destructive, the 


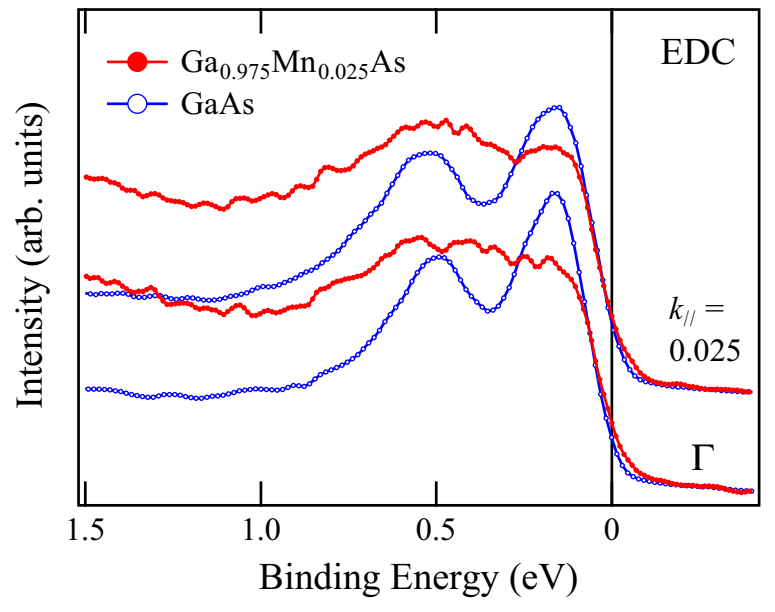

FIG. 2. (Color online) Comparison of EDCs in vicinity of the $\Gamma$ point taken at $h v=890 \mathrm{eV}$ between $\mathrm{Ga}_{0.975} \mathrm{Mn}_{0.025} \mathrm{As}$ and $\mathrm{GaAs}$ thin films. The EDCs of GaAs are shifted to match the band dispersion to that of $\mathrm{Ga}_{0.975} \mathrm{Mn}_{0.025}$ As. The momentum $k_{\|}$is along the $\Gamma-\mathrm{K}-\mathrm{X}$ direction and its unit is $2 \sqrt{2} \pi / a$, where $a$ is the in-plane lattice constant. $(\mathrm{Ga}, \mathrm{Mn})$ As shows additional spectral weight at $E_{\mathrm{F}}$ consistent with its metallic conductivity.

electronic structure near the surface in these experiments may be different from the bulk. Even in the case of in situ photoemission on $(\mathrm{Ga}, \mathrm{Mn}) \mathrm{As}$ [28], the carrier concentration in the vicinity of the surface is possibly suppressed from the bulk one. This observation warrants that the SX-ARPES measurements with their enhanced probing depth reflect the bulk electronic structure of $(\mathrm{Ga}, \mathrm{Mn}) \mathrm{As}$. No signal from the Mn $3 d$ states can be distinguished from the VB of host GaAs in these spectra taken at high $h v$.

It is important to mention a negligible contribution of the band bending near the interface between the amorphous As overlayer and $(\mathrm{Ga}, \mathrm{Mn}) \mathrm{As}$ underlayer. The bending, if it existed, would strongly influence the interpretation of the experimental data described below. Figure 3 compares the band dispersion between different BZs across the $k_{z}$ direction. The ARPES image was taken at $h v=890 \mathrm{eV}$ (the seventh BZ). The superimposed points mark peak positions of the momentum distribution curves (MDCs) in the ARPES image taken at $h v=655 \mathrm{eV}$ (the sixth BZ). Both the dispersive bands look identical, as shown in Fig. 3. Taking into account the increase of the photoelectron mean free path $\lambda$ with $h v$, this result demonstrates that the band bending near the interface between the (Ga,Mn)As underlayer and amorphous As overlayer is negligible. Additionally, the observation that difference of As $3 d$ core-level peak positions between the surface and the bulk components (see Fig. S2 [23]) is constant as a function of $h v$ suggests the negligible contribution of the band bending near the interface. These results imply that even in the vicinity of the interface, $E_{\mathrm{F}}$ is located in the same position of the bulk $(\mathrm{Ga}, \mathrm{Mn})$ As underlayer.

\section{B. Mn 3d-derived impurity band}

The energy position of the Mn $3 d$ states in VB has intensively been debated for more than a decade. To address this issue, we have conducted resonant angle-resolved

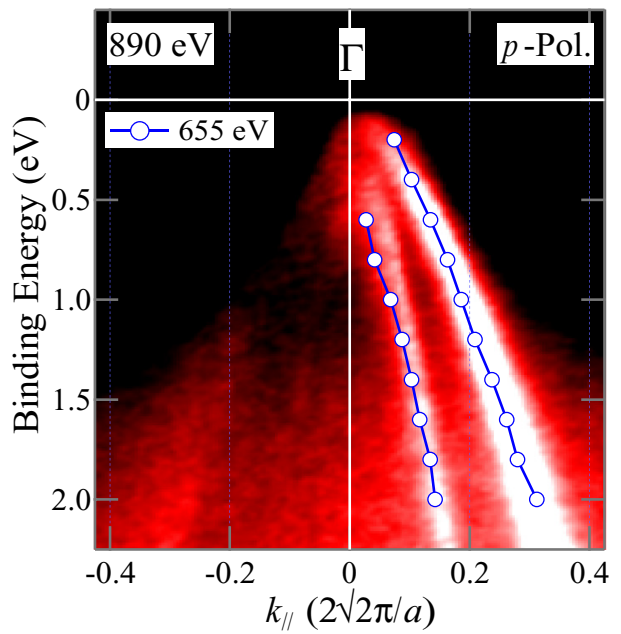

FIG. 3. (Color online) Comparison of the band dispersions between different BZs across $k_{z}$. The momentum $k_{\|}$is along the $\Gamma-\mathrm{K}-\mathrm{X}$ direction. The band dispersions taken at $h v=650 \mathrm{eV}$ are determined by fitting the experimental MDCs with the Lorentzian function. The identity of the dispersions measured with different probing depths excludes any significant band bending.

photoemission spectroscopy (r-ARPES) at the Mn $L_{3}$ absorption edge. r-ARPES is known to probe the element-specific band dispersion of open-shell $d$ or $f$ electron systems [30,31]. Figure 4(a) shows the XAS spectrum at the Mn $L_{2,3}$ edges of the sample. Previously, the magnetic field dependence of $\mathrm{x}$-ray magnetic circular dichroism (XMCD) has demonstrated that $(\mathrm{Ga}, \mathrm{Mn})$ As includes two kinds of Mn components: the intrinsic one, which is most likely due to ferromagnetic substitutional $\mathrm{Mn}$ ions, including some of the paramagnetic interstitial $\mathrm{Mn}^{2+}$ ions, and the extrinsic one, which is due to paramagnetic oxidized $\mathrm{Mn}^{2+}$ ions segregated in the surface region $[17,32]$. The XAS peak position of the ferromagnetic intrinsic Mn component of $640 \mathrm{eV}$ is different from that of the paramagnetic extrinsic component of $640.5 \mathrm{eV}$ [red and blue vertical lines in Fig. 4(a)] [17]. Accordingly, the resonance enhancement of ARPES measured at $h v=640 \mathrm{eV}$ should be relevant to the intrinsic components, while the r-ARPES spectrum taken at $h v=640.5 \mathrm{eV}$ should have almost zero contribution of the ferromagnetic states. Therefore a comparison between the r-ARPES spectra taken at 640 and $640.5 \mathrm{eV}$ will reveal the Mn $3 d$ states of the intrinsic origin which are responsible for the FM.

Figures 4(c)-4(f) show a series of r-ARPES images measured on our $(\mathrm{Ga}, \mathrm{Mn})$ As sample under variation of $h v$ with $s$ polarization. [No shift of the band dispersion could be seen in the spectra taken at different BZs (see Fig. 3).] Most important to note in the evolution of the r-ARPES images is the $\mathrm{Mn}$ $3 d$-derived IB which pops up at $h v=640 \mathrm{eV}$ in the vicinity of $E_{\mathrm{F}}$, as shown in Fig. 4(d), due to the resonance enhancement at the excitation energy of the ferromagnetic intrinsic Mn component, not paramagnetic extrinsic Mn oxides. The resonance at $h v=640 \mathrm{eV}$ also enhances the intensity around $E_{B} \sim 3 \mathrm{eV}$, which is due to the well-known main peak of the Mn $3 d$ partial density of states [29]. Conversely, the r-ARPES image in Fig. 4(e), taken at $h v=640.5 \mathrm{eV}$, which resonates with the paramagnetic Mn component and is nonresonant for the 

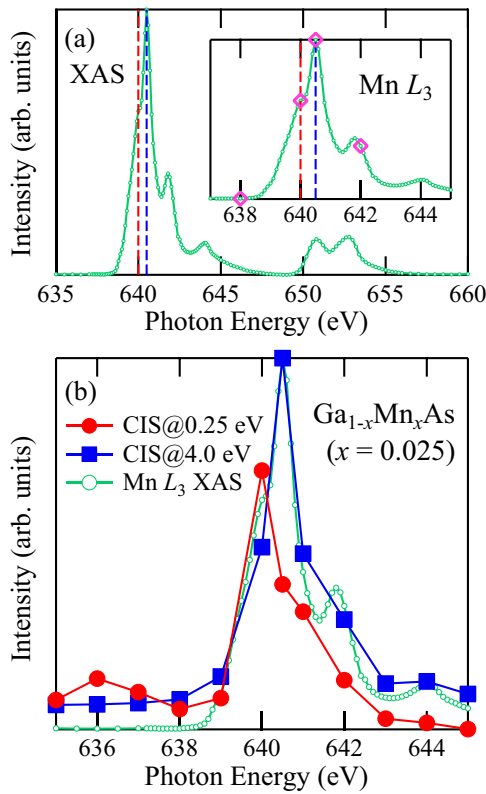
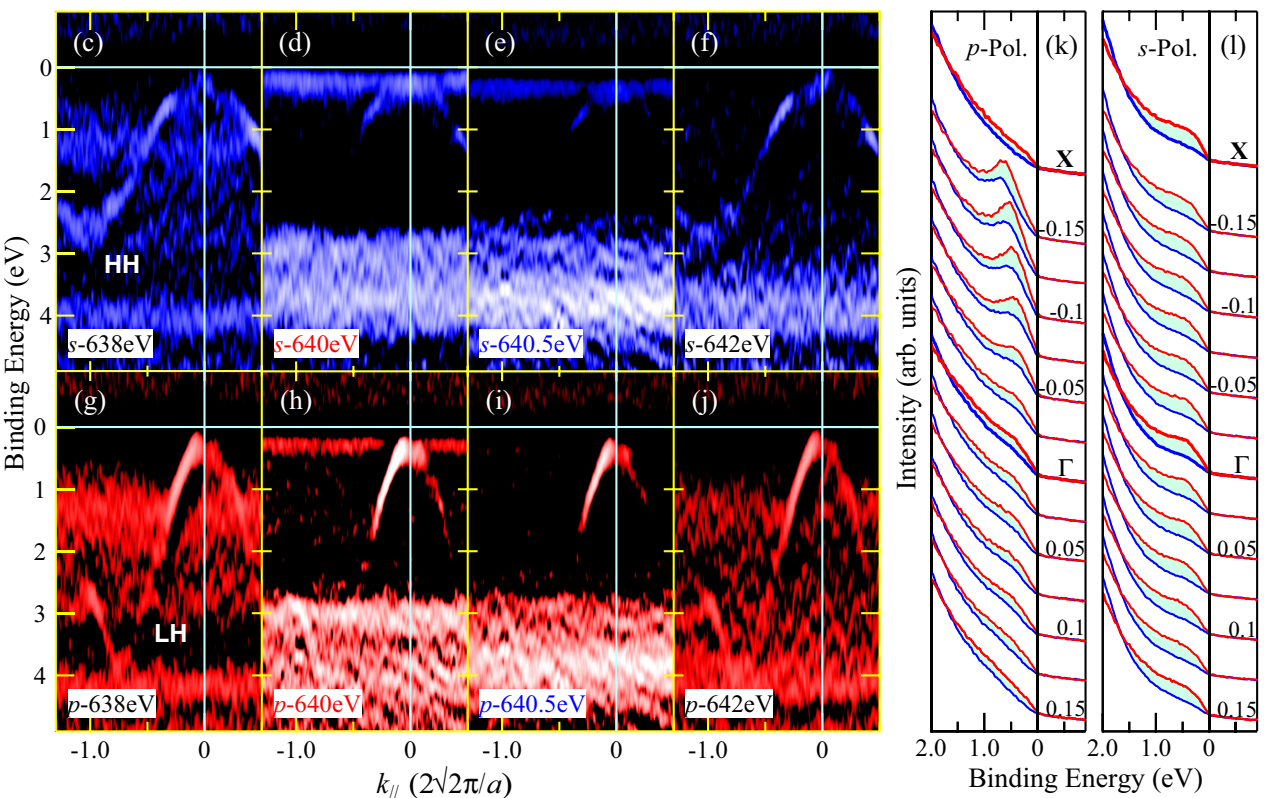

FIG. 4. (Color online) Resonant angle-resolved photoemission spectroscopy (r-ARPES) spectra at the $\mathrm{Mn}_{2,3}$ edge in $\mathrm{Ga}_{0.975} \mathrm{Mn}_{0.025} \mathrm{As}$. (a) Mn $L_{2,3}$ XAS spectrum. The red and blue vertical dashed lines correspond to the resonant $h v$ for the ferromagnetic and paramagnetic components, respectively. (b) Constant-initial-state (CIS) spectra taken at $E_{B}=0.25$ and $4.0 \mathrm{eV}$ with $s$ polarization. (c)-(f), (g)-(j) r-ARPES images taken in a series of at $h v$ from 638 to $642 \mathrm{eV}$ with $s$ and $p$ polarization, respectively, with the $h v$ points shown as rhombi in the inset of (a). All the images are represented in second derivative along $E_{B}$ (positive values of $-d^{2} I / d E^{2}$ [42]) and plotted in logarithmic scale to emphasize the resonance enhancements. $(\mathrm{k}, 1)$ Resonant EDCs around the $\Gamma$ and at the $X$ points taken with $p$ and $s$ polarizations, respectively. The red curves are the EDCs for $h v=640 \mathrm{eV}$, the blue ones for $h v=640.5 \mathrm{eV}$. The green areas denote differences between the red and blue curves. These linear dichroism results identify the IB hybridization with the LH band of GaAs but not with the HH band.

intrinsic component, shows only an afterglow of the IB near $E_{\mathrm{F}}$ and another nondispersive resonant projection occurs around $E_{B} \sim 4 \mathrm{eV}$. Furthermore, Fig. 4(b) shows angle-integrated photoemission intensity at fixed $E_{B}=0.25 \mathrm{eV}$ (where the photoemission intensity blows up at the ferromagnetic XAS peak) and $4.0 \mathrm{eV}$ (at the paramagnetic XAS peak) evaluated as a function of $h v$, the so-called constant-initial-state (CIS) spectrum. Consistently with the above ARPES images, these spectra show well-defined resonant peaks corresponding to the Fano profile [33]. The clear energy separation of the peaks in the two CIS spectra indicates that the ferromagnetic component resonates at $h v$ different from the paramagnetic component.

We note that the existence of the IB has been reported in previous ARPES measurements on (Ga,Mn)As with vacuumultraviolet rays ( $18 \leqq h v \leqq 40 \mathrm{eV})$ [10] and hard $\mathrm{x}$ rays [11], but the location of the IB was deeper $\left(\sim 0.4 \mathrm{eV}\right.$ below $\left.E_{\mathrm{F}}\right)$ than in our observation and overlapped with the VBM. Furthermore, theoretical calculations subsequent to the vacuum-ultraviolet ARPES [10] suggested that the IB originated from the interstitial Mn impurities [34]. The present observations give unambiguous evidence that the IB is in fact located near the VBM and formed by the intrinsic Mn ions directly related to the FM in $(\mathrm{Ga}, \mathrm{Mn}) \mathrm{As}$. Even though the intrinsic Mn components include not only the Mn ions substituting for the Ga site, but also partly interstitial Mn ions antiferromagnetically coupled to the substitutional Mn, influence of the interstitial $\mathrm{Mn}$ is minor in the r-ARPES spectra because the amount of the interstitial $\mathrm{Mn}$ ions is only $\sim 10 \%$ of the total amount of $\mathrm{Mn}$ ions in $(\mathrm{Ga}, \mathrm{Mn}) \mathrm{As}$ [35]. We note that although the spectral weight seen at $E_{\mathrm{F}}$ is small, it must be nonzero because of its disordered character of the IB, which introduces energy broadening of this band with its high-energy tail approaching $E_{\mathrm{F}}$.

In addition, incident light polarization dependence of the r-ARPES signal sheds light on details of the hybridization between the Mn $3 d$ orbital and the ligand bands. Figures 4(g)-4(j) show the Mn $L_{3}$ r-ARPES series of the (Ga,Mn)As sample taken with $p$ polarization. The offresonance image taken at $h v=638 \mathrm{eV}$ shows the $\mathrm{LH}$ band of host GaAs over the background of nondispersive amorphous As states, as shown in Fig. 4(g). The $h v=640 \mathrm{eV}$ resonance of the ferromagnetic intrinsic Mn component enhances the $\mathrm{Mn}$ $3 d$-derived IB in the vicinity of the VB maximum and the $\mathrm{Mn}$ $3 d$ partial density of states around binding energy $E_{B} \sim 3 \mathrm{eV}$, as shown in Fig. 4(h). It is important to note here that together with the IB the $h v=640 \mathrm{eV}$ resonance also pops up the LH band. These manifest the IB hybridization with the LH band. In contrast, the ARPES images shown in Figs. 4(c)-4(f), taken with $s$ polarization, which exposes the $\mathrm{HH}$ band, do not show any increase of the $\mathrm{HH}$ intensity on top of the IB, suggesting its insignificant hybridization with the $\mathrm{HH}$ band. The results indicate that the IB hybridizes with the LH band but not with the $\mathrm{HH}$ band, having a different wave-function character. These conclusions are confirmed by Figs. 4(k) and 4(1), which show the linear-polarization dependence of the EDCs around the $\Gamma$ and at the X points measured at the Mn $L_{3}$ resonance. With $p$ polarization, which exposes the $\mathrm{LH}$ 


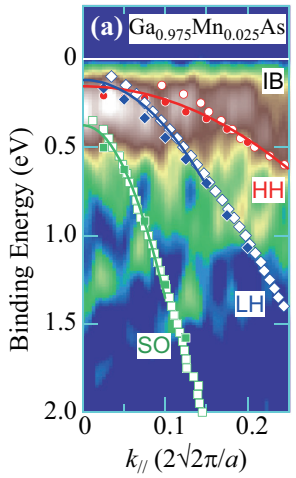

(b)

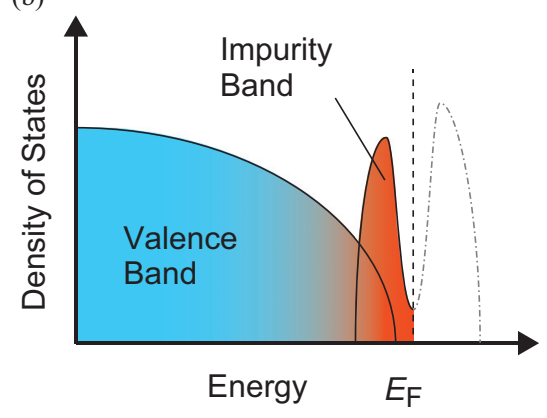

FIG. 5. (Color online) Valence-band structure of (Ga,Mn)As. (a) Band dispersion around the $\Gamma$ point near $E_{\mathrm{F}}$ along the $\Gamma-\mathrm{K}-\mathrm{X}$ line. IB is the Mn $3 d$-derived impurity band on top of the $\mathrm{LH}, \mathrm{HH}$, and SO bands of GaAs. Open and closed symbols are the MDC and EDC peak positions, respectively, for the GaAs bands fitted with a quadratic polynomial. The solid curves are their fit with a quadratic polynomial function centered at the $\Gamma$ point to the band dispersion. (b) The experimental band diagram as densities of states of the Mn $3 d$-derived IB embedded into the VB in host GaAs. Dash-dotted line denotes anticipated unoccupied density of states.

band of host GaAs, the difference of the EDCs taken at 640 and $640.5 \mathrm{eV}$ [the green area in Fig. 4(k)], representing the resonant contribution, disperses as a function of $k$, closely following the LH band. As opposed to the $p$ polarization, the EDCs taken with $s$ polarization, which exposes the HH band, show only the $k$-independent intensity of the IB, confirming the insignificant hybridization between the IB and HH band. Furthermore, a close look at the resonant tunneling results [14] shows that with incorporation of Mn the LH resonant tunneling peak displaces and the HH peak stays at the same energy. This is fully consistent with our r-ARPES results, which unambiguously identify the hybridization of the Mn IB with the LH GaAs band and only its insignificant hybridization with the $\mathrm{HH}$ band.

In addition to the formation of IB, the doped Mn atoms into $\mathrm{GaAs}$ also disorder the $\mathrm{GaAs}$ lattice, resulting in broadening of the GaAs bands as shown in Fig. 2. This means that although the GaAs bands terminate below $E_{\mathrm{F}}$, because of their energy broadening there should be finite GaAs weight at $E_{\mathrm{F}}$, which can in principle contribute to the conductivity. Qualitatively, the disordered GaAs may suppress the carrier-induced ferromagnetism in GaMnAs because the spin information of carriers is smeared by scattering on the GaAs defects. However, competition between the IB and disordered GaAs states may be relevant only for much larger Mn concentrations close to the optimal. Quantitative details regarding the disorder contribution will be addressed in further research of the band dispersion near $E_{\mathrm{F}}$ with different $\mathrm{Mn}$ concentrations.

\section{Valence-band structure and origin of ferromagnetism in $(\mathbf{G a}, \mathbf{M n}) \mathrm{As}$}

Figure 5(a) shows a summary of the VB structure of $(\mathrm{Ga}, \mathrm{Mn}) \mathrm{As}$ near the $\Gamma$ point. Here, the positions of the $\mathrm{LH}, \mathrm{SO}$, and $\mathrm{HH}$ bands were estimated from the Lorentzian (a)

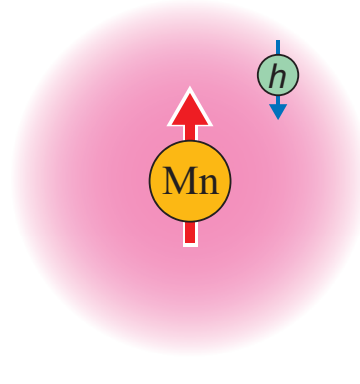

(b)

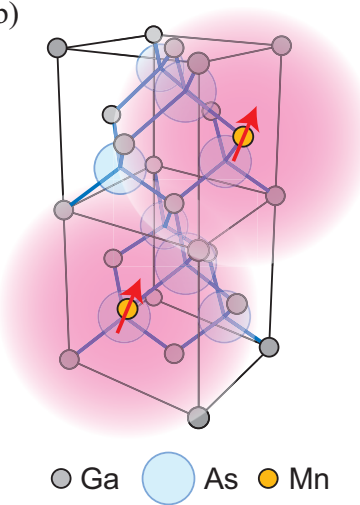

FIG. 6. (Color online) Bound magnetic polarion picture of FM in (Ga,Mn)As. (a) Zhang-Rice singlet-like bound magnetic polaron around the doped Mn ion. (b) Schematic image of overlap among the magnetic polarons in $(\mathrm{Ga}, \mathrm{Mn}) \mathrm{As}$.

fits of MDCs of the experimental ARPES images. The obtained dispersive bands were fitted in turn with quadratic polynomial functions. The estimated positions of the LH, $\mathrm{HH}$, and SO band maxima are at $E_{B}=119 \pm 44 \mathrm{meV}$, $155 \pm 36 \mathrm{meV}$, and $376 \pm 24 \mathrm{meV}$, respectively, providing us with further evidence that VB of the host GaAs is located below $E_{\mathrm{F}}$.

Figure 5(b) embeds our main experimental findings in a schematic diagram of the VB electronic structure of (Ga,Mn)As: I. Formation of a nondispersive Mn $3 d$-derived IB is mostly related to the substitutional Mn impurities. The absence of its dispersion demonstrates the random distribution of the $\mathrm{Mn}$ ions in $(\mathrm{Ga}, \mathrm{Mn}) \mathrm{As}$, with the crystal momentum $\mathbf{k}$ being not a good quantum number for this band. Therefore, the formation of the IB responsible for the FM can be theoretically understood in the framework of local models. The center of the occupied part of the IB resulting from carrier compensation is located below $E_{\mathrm{F}}$, while the majority of the IB should be located above $E_{\mathrm{F}}$. The IB spectral weight depletes as it approaches $E_{\mathrm{F}}$, implying the incoherent nature of charge carriers. II. The VBM of host GaAs is also located below $E_{\mathrm{F}}$. III. The Mn $3 d$ band shows $k$-independent hybridization with the VB, specifically, with the LH band. Although such low Mn concentration of $(\mathrm{Ga}, \mathrm{Mn}) \mathrm{As}$ is near the onset of FM $[8,9]$, our preliminary SX-ARPES results on $(\mathrm{Ga}, \mathrm{Mn})$ As with the $\mathrm{Mn}$ concentration up to $10 \%$ have shown qualitatively the same general features of the VB with almost the same energy position of the nondispersive IB.

Our picture of the IB formation and the associated FM in $(\mathrm{Ga}, \mathrm{Mn}) \mathrm{As}$ may be naturally understood starting from the Anderson impurity model (AIM) [36], which describes the transition-metal $d$ orbitals and the host band electrons hybridizing with each other. When hybridization between the $d$ orbital and the host ligand band is strong enough, this model predicts the formation of a split-off Mn-impurity state located above VBM for a Mn ion embedded in GaAs host [10], where the Mn ion weakly bounds to a hole carrier. (Details of the derivation are given in the Appendix.) The schematic image is shown in Fig. 6(a). This picture is analogous to the Zhang-Rice singlet (ZRS) state in high- $T_{c}$ cuprates [37] and well explains the binding energy of the Mn acceptor level in 
GaAs:Mn (Mn concentration of $1.1 \times 10^{17} \mathrm{~cm}^{-3}$ ), $\sim 110 \mathrm{meV}$ above VBM estimated from luminescence and electron spin resonance measurements [38,39].

According to the percolation theory of BMPs, the nonmonotonic temperature dependence of the transport properties of $(\mathrm{Ga}, \mathrm{Mn}) \mathrm{As}$ is qualitatively explained [16] as due to the hole localization around the Mn ions. From cluster-model calculations of the Mn $2 p$ core-level photoemission spectra [40], the exchange interaction between the $d$ magnetic moment and carrier spin for the split-off state $N \beta$ has previously been estimated as $-1.2 \pm 0.2 \mathrm{eV}$, where a negative sign means antiferromagnetic magnetic interaction between the $3 d$ moment and carrier spin. In this case, most of Mn $3 d$ character hybridized into the VB goes to the split-off state and forms the IB, and the exchange splitting of the host GaAs band remains very weak, as observed by the resonant tunneling study $[13,14]$. The local-density approximation calculations have predicted the existence of the IB as the antibonding states arising from the hybridization between the Mn $3 d$ state and a ligand band [7], in contrast to the spin splitting of VB which is expressed as $\sim x N \beta\langle S\rangle$ in the picture of mean-field theory $[3,4]$. Following these arguments, we conjecture that the formation of the IB is derived from the split-off state resulting in the ZRS-like BMP, with an overlap among the BMPs aligning the magnetic moments of the Mn ions in (Ga,Mn)As [Fig. 6(b)], and therefore the percolation of BMPs is the origin of the carrier-related FM in (Ga,Mn)As.

\section{CONCLUSION}

We have performed SX-ARPES measurements on the magnetic semiconductor (Ga,Mn)As in order to unveil the valence-band structure, in particular, regarding the existence of the Mn $3 d$-derived IB and its hybridization with host GaAs bands. Using SX-ARPES with its enhanced penetrating ability, we have accessed the three-dimensional bulk valenceband structure in (Ga,Mn)As through a passivation layer of amorphous As. The experimental dispersions of the host GaAs (the $\mathrm{LH}, \mathrm{HH}$, and $\mathrm{SO}$ bands) around the $\Gamma$ point indicate that the VBM of the host GaAs bands stays below $E_{\mathrm{F}}$. Furthermore, resonant ARPES at the $\mathrm{Mn} L_{3}$ absorption edge reveals that the nondispersive Mn 3d-derived IB responsible for the FM is located in the vicinity of the VBM and is hybridized with the GaAs ligand bands, specifically with the LH band. The band diagram emerging from these experimental findings combines and details the previous double-exchange and $p$ - $d$ exchange models of the FM in $(\mathrm{Ga}, \mathrm{Mn})$ As. The dispersionless character of the Mn 3d-derived IB unveils its origin as the split-off $M n$ impurity state predicted by the AIM. This suggests formation of the ZRS-like BMPs, whose overlap aligns the magnetic moments of the Mn ions to stabilize the FM of (Ga,Mn)As. Therefore, the origin of the carrier-induced FM in (Ga,Mn)As appears predominantly due to the percolation of BMPs. Generalizing our methodology, we have demonstrated that SX-ARPES, with its enhanced probing depth and elemental and chemical state, and specificity achieved with resonant photoemission, can successfully unveil details of the electronic structure of DMS systems. The promised physical information on the physics of DMS systems will deepen the fundamental understanding of carrier-induced FM and help development of future spintronic device applications.

\section{ACKNOWLEDGMENTS}

The authors thank C. Fadley, J. Minár, and J. Kanski for informative discussions. This work was supported by a Grantin-Aid for Scientific Research (Grants No. S22224005 and No. 23000010) from JSPS, Japan. The authors thank C. Quitmann, F. van der Veen, and J. Mesot for their continuous support of the SX-ARPES project at SLS. M.K. acknowledges support from the Japan Society for the Promotion of Science.

\section{APPENDIX: SPLIT-OFF MN-IMPURITY STATE INDUCED BY STRONG $\boldsymbol{p}$ - $d$ HYBRIDIZATION}

A single transition-metal or rare-earth ion embedded in an infinite lattice of nonmetal ions can be described by the Anderson impurity model [36]. In AIM, the band electron state $\psi_{\varepsilon q}$, which has the same symmetry group $\gamma$ and spin $\sigma$ as the $d$ electron state $\psi_{q}(q \equiv \gamma \sigma)$, is hybridized with $\psi_{q}$ [41]. These states are assumed to be orthonormalized:

$$
\left\langle\varepsilon q \mid \varepsilon^{\prime} q^{\prime}\right\rangle=\delta\left(\varepsilon-\varepsilon^{\prime}\right) \delta_{q, q^{\prime}}, \quad\left\langle q \mid q^{\prime}\right\rangle=\delta_{q, q^{\prime}}, \quad\left\langle\varepsilon q \mid q^{\prime}\right\rangle=0 .
$$

Using these one-electron orbital bases, the Hamiltonian of the AIM is given by

$$
\begin{aligned}
H & =H_{d}+H_{p}+H_{p d}, \\
H_{d} & =\sum_{q=1}^{10} \varepsilon_{d q}^{0} n_{q}+\frac{1}{2} \sum_{q, q^{\prime}, q^{\prime \prime}, q^{\prime \prime \prime}=1}^{10}\left\langle q q^{\prime}|\nu| q^{\prime \prime} q^{\prime \prime \prime}\right\rangle c_{q^{\prime}}^{\dagger} c_{q}^{\dagger} c_{q^{\prime \prime}} c_{q^{\prime \prime \prime}}, \\
H_{p} & =\sum_{q=1}^{10} \int_{\varepsilon_{p}-W_{p} / 2}^{\varepsilon_{p}+W_{p} / 2} d \varepsilon \varepsilon n_{\varepsilon q}, \\
H_{p d} & =-\sum_{q=1}^{10} \int_{\varepsilon_{p}-W_{p} / 2}^{\varepsilon_{p}+W_{p} / 2} d \varepsilon\left[t_{\varepsilon q} c_{\varepsilon q}^{\dagger} c_{q}+t_{\varepsilon q}^{*} c_{q}^{\dagger} c_{\varepsilon q}\right],
\end{aligned}
$$

where $W_{p}$ is the bandwidth of the ligand-orbital-derived VB centered at $\varepsilon_{p}, c_{\varepsilon q}^{\dagger}(c \varepsilon q)$ is the creation (annihilation) operator of the band electron, and $t_{\varepsilon q}$ is the transfer integral.

In AIM, the wave function of the one-electron state is a linear combination of the $d$ and valence-band states as

$$
\tilde{\psi}_{q}=a_{q}\left[\psi_{q}+\int_{\varepsilon_{p}-W_{p} / 2}^{\varepsilon_{p}+W_{p} / 2} d \varepsilon^{\prime} c_{q}\left(\varepsilon^{\prime}\right) \psi_{\varepsilon^{\prime} q}\right] .
$$

Here, $a_{q}$ is the normalization constant. The matrix elements of the Hartree-Fock operator are

$$
\begin{aligned}
\left\langle\gamma_{\downarrow}\left|h^{\mathrm{HF}}\right| \gamma_{\downarrow}\right\rangle & =\varepsilon_{d}^{0}+4 U^{\prime}-4 J_{H} \equiv \varepsilon_{d}^{\prime \prime}, \\
\left\langle\varepsilon_{\gamma_{\downarrow}}\left|h^{\mathrm{HF}}\right| \varepsilon_{\gamma_{\downarrow}}^{\prime}\right\rangle & =\varepsilon \delta\left(\varepsilon-\varepsilon^{\prime}\right), \\
\left\langle\varepsilon_{\gamma_{\downarrow}}\left|h^{\mathrm{HF}}\right| \gamma_{\downarrow}\right\rangle & =-t_{\varepsilon \gamma} .
\end{aligned}
$$

The Hartree-Fock equation for the state (A3) to be solved is given by

$$
\left(h^{\mathrm{HF}}-\varepsilon\right) \tilde{\psi}_{\gamma \downarrow}=0 .
$$




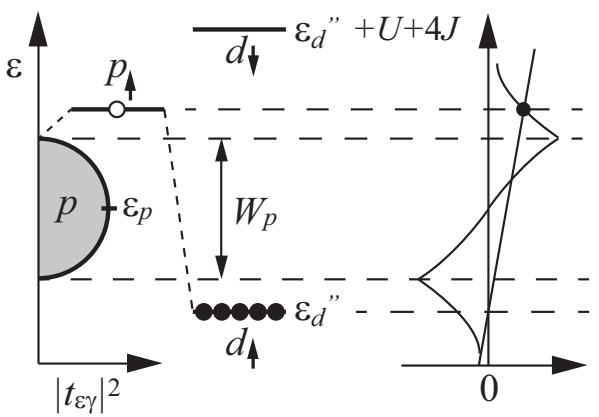

FIG. 7. One-electron levels of AIM for the Mn ion in (Ga,Mn)As showing a split-off state above the VBM. Left: Strength of the $p$-d hybridization $\left|t_{\varepsilon^{\prime} \gamma}\right|^{2}$. Right: The right side and left side of the integral equation (A9) as a function of $\varepsilon$. The crossing point indicated by the black circle gives the solution for the discrete level (split-off state) occupied by the doped hole.

By multiplying $\psi_{\gamma \downarrow}^{*}$ to Eq. (A4) and then integrating through the VB, the following equation is obtained:

$$
\left(\varepsilon_{d}^{\prime \prime}-\varepsilon\right)-\int_{\varepsilon_{p}-W_{p} / 2}^{\varepsilon_{p}+W_{p} / 2} d \varepsilon^{\prime} c_{\gamma_{\downarrow}}\left(\varepsilon^{\prime}\right) t_{\varepsilon^{\prime} \gamma}^{*}=0 .
$$

Additionally, by multiplying $\psi_{\varepsilon \gamma \downarrow}^{*}$ to Eq. (A4) and then integrating through the $\mathrm{VB}$, another equation is obtained:

$$
\begin{gathered}
-t_{\varepsilon^{\prime} \gamma}+c_{\gamma_{\downarrow}}\left(\varepsilon^{\prime}\right)\left(\varepsilon^{\prime}-\varepsilon\right)=0, \\
\therefore c_{\gamma_{\downarrow}}\left(\varepsilon^{\prime}\right)=\frac{t_{\varepsilon^{\prime} \gamma}}{\varepsilon^{\prime}-\varepsilon} .
\end{gathered}
$$

Then the integral equation for $c_{\gamma_{\downarrow}}\left(\varepsilon^{\prime}\right)$ is given by substituting Eq. (A8) in Eq. (A6):

$$
\int_{\varepsilon_{p}-W_{p} / 2}^{\varepsilon_{p}+W_{p} / 2} d \varepsilon^{\prime} \frac{\left|t_{\varepsilon^{\prime} \gamma}\right|^{2}}{\varepsilon^{\prime}-\varepsilon}=\varepsilon-\varepsilon_{d}^{\prime}
$$

Here, $\gamma$ is the $t_{2}$ level, i.e., $\gamma=d_{x y}, d_{y z}, d_{z x}$. For simplicity, $\left|t_{\varepsilon^{\prime} \gamma}\right|^{2}$ is assumed to be a semiellipsoid within $\varepsilon_{p}-W_{p} / 2<$ $\varepsilon<\varepsilon_{p}+W_{p} / 2$, as shown in the left panel of Fig. 7. The left side of Eq. (A9) has singularities at the edges of the VB $\left(\varepsilon=\varepsilon_{p} \pm W_{p} / 2\right)$, as shown in the right panel of Fig. 7. If the hybridization is strong enough, a crossing point exists between the left side and the right side of Eq. (A9) outside of the VB continuum, and a discrete level, i.e., a split-off state is formed above the VB maximum through the hybridization, as shown in Fig. 7.

Let us consider the electronic structure of a single $\mathrm{Mn}$ ion in $(\mathrm{Ga}, \mathrm{Mn}) \mathrm{As}$. If the $\mathrm{Mn}$ ion substituting for the $\mathrm{Ga}^{3+}$ site is trivalent $\mathrm{Mn}^{3+}$, the charge neutrality condition is satisfied. In contrast, if the $\mathrm{Mn}$ ion is divalent $\mathrm{Mn}^{2+}$, the ion supplies a hole to the VB to satisfy the charge neutrality condition. Because the hole should have the down-spin, the $d$ spin strongly couples with the spin of the $p$ hole antiferromagnetically. Taking into account the configuration interaction method, the same system can be described from a many-electrons point of view. Assuming that the many-electron ground state $\Psi_{N}^{\mathrm{CI}}$ is expanded by wave functions $\Psi\left(d^{4}\right)$ and $\Psi\left(d^{5} \underline{\varepsilon}\right)$, which represent the electronic configurations $d^{4}$ and $d^{5} \underline{\varepsilon}$, an integral equation similar to Eq. (A9) is obtained, where $\underline{\varepsilon}$ denotes a hole in the ligand. Thereby, the discrete split-off state appears below the continuum $d_{\uparrow}^{5} \underline{\varepsilon}_{\downarrow}$ as the solution of the integral equation, as well as the one-electron states [41].
[1] T. Dietl, Nature Mater. 9, 965 (2010).

[2] T. Jungwirth, J. Sinova, J. Mašek, J. Kučera, and A. H. MacDonald, Rev. Mod. Phys. 78, 809 (2006).

[3] T. Dietl, H. Ohno, F. Matsukura, J. Cibert, and D. Ferrand, Science 287, 1019 (2000).

[4] T. Jungwirth, J. Sinova, A. H. MacDonald, B. L. Gallagher, V. Novak, K. W. Edmonds, A. W. Rushforth, R. P. Campion, C. T. Foxon, L. Eaves, E. Olejnik, J. Masek, S. R. Eric Yang, J. Wunderlich, C. Gould, L. W. Molenkamp, T. Dietl, and H. Ohno, Phys. Rev. B 76, 125206 (2007).

[5] M. Sawicki, D. Chiba, A. Korbecka, Y. Nishitani, J. A. Majewski, F. Matsukura, T. Dietl, and H. Ohno, Nat. Phys. 6, 22 (2010).

[6] M. Berciu and R. N. Bhatt, Phys. Rev. Lett. 87, 107203 (2001).

[7] P. Mahadevan and A. Zunger, Appl. Phys. Lett. 85, 2860 (2004).

[8] B. L. Sheu, R. C. Myers, J.-M. Tang, N. Samarth, D. D. Awschalom, P. Schiffer, and M. E. Flatté, Phys. Rev. Lett. 99, 227205 (2007).

[9] B. C. Chapler, R. C. Myers, S. Mack, A. Frenzel, B. C. Pursley, K. S. Burch, E. J. Singley, A. M. Dattelbaum, N. Samarth, D. D. Awschalom, and D. N. Basov, Phys. Rev. B 84, 081203 (2011).
[10] J. Okabayashi, A. Kimura, O. Rader, T. Mizokawa, A. Fujimori, T. Hayashi, and M. Tanaka, Phys. Rev. B 64, 125304 (2001).

[11] A. X. Gray, J. Minár, P. R. Stone, Y. Yamashita, J. Fujii, J. Braun, L. Plucinski, C. M. Schneider, G. Panaccione, H. Ebert, O. D. Dubon, K. Kobayashi, and C. S. Fadley, Nat. Mater. 11, 957 (2012).

[12] K. S. Burch, D. B. Shrekenhamer, E. J. Singley, J. Stephens, B. L. Sheu, R. K. Kawakami, P. Schiffer, N. Samarth, D. D. Awschalom, and D. N. Basov, Phys. Rev. Lett. 97, 087208 (2006).

[13] S. Ohya, K. Takata, and M. Tanaka, Nat. Phys. 7, 342 (2011).

[14] S. Ohya, I. Muneta, P. N. Hai, and M. Tanaka, Phys. Rev. Lett. 104, 167204 (2010).

[15] A. Kaminski and S. Das Sarma, Phys. Rev. Lett. 88, 247202 (2002).

[16] A. Kaminski and S. Das Sarma, Phys. Rev. B 68, 235210 (2003).

[17] Y. Takeda, M. Kobayashi, T. Okane, T. Ohkochi, J. Okamoto, Y. Saitoh, K. Kobayashi, H. Yamagami, A. Fujimori, A. Tanaka, J. Okabayashi, M. Oshima, S. Ohya, P. N. Hai, and M. Tanaka, Phys. Rev. Lett. 100, 247202 (2008).

[18] C. S. Fadley, Synchrotron Radiat. News 25, 26 (2012). 
[19] V. N. Strocov, M. Kobayashi, X. Wang, L. L. Lev, J. Krempasky, V. V. Rogalev, T. Schmitt, C. Cancellieri, and M. L. ReinleSchmitt, Synchrotron Radiat. News 27, 31 (2014).

[20] V. N. Strocov, T. Schmitt, U. Flechsig, T. Schmidt, A. Imhof, Q. Chen, J. Raabe, R. Vetemps, D. Zimoch, J. Krempasky, X. Wang, M. Grioni, A. Piazzalunga, and L. Patthey, J. Synchrotron Rad. 17, 631 (2010).

[21] V. N. Strocov, X. Wang, M. Shi, M. Kobayashi, J. Krempasky, C. Hess, T. Schmitt, and L. Patthey, J. Synchrotron Rad. 21, 32 (2014).

[22] N. J. Kawai, T. Nakagawa, T. Kojima, K. Ohta, and M. Kawashima, Eelectron. Lett. 20, 47 (1984).

[23] See Supplemental Material at http://link.aps.org/supplemental/ 10.1103/PhysRevB.89.205204 for the explanation of SXARPES through an amorphous capping layer and a movie of constant energy mapping as a function of binding energy.

[24] M. Kobayashi, I. Muneta, T. Schmitt, L. Patthey, S. Ohya, M. Tanaka, M. Oshima, and V. N. Strocov, Appl. Phys. Lett. 101, 242103 (2012)

[25] T.-C. Chiang, J. A. Knapp, M. Aono, and D. E. Eastman, Phys. Rev. B 21, 3513 (1980).

[26] J.-W. Luo, G. Bester, and A. Zunger, Phys. Rev. Lett. 102, 056405 (2009).

[27] A. X. Gray, C. Papp, S. Ueda, B. Balke, Y. Yamashita, L. Plucinski, J. Minár, J. Braun, E. T. Ylvisaker, C. M. Schneider, W. E. Pickett, H. Ebert, K. Kobayashi, and C. S. Fadley, Nat. Mater. 10, 759 (2011).

[28] H. Åsklund, L. Ilver, J. Kanski, J. Sadowski, and R. Mathieu, Phys. Rev. B 66, 115319 (2002).

[29] O. Rader, C. Pampuch, A. M. Shikin, W. Gudat, J. Okabayashi, T. Mizokawa, A. Fujimori, T. Hayashi, M. Tanaka, A. Tanaka, and A. Kimura, Phys. Rev. B 69, 075202 (2004).
[30] H. J. Im, T. Ito, H.-D. Kim, S. Kimura, K. E. Lee, J. B. Hong, Y. S. Kwon, A. Yasui, and H. Yamagami, Phys. Rev. Lett. 100, 176402 (2008).

[31] M. Mulazzi, A. Chainani, N. Katayama, R. Eguchi, M. Matsunami, H. Ohashi, Y. Senba, M. Nohara, M. Uchida, H. Takagi, and S. Shin, Phys. Rev. B 82, 075130 (2010).

[32] K. W. Edmonds, P. Boguslawski, K. Y. Wang, R. P. Campion, S. N. Novikov, N. R. S. Farley, B. L. Gallagher, C. T. Foxon, M. Sawicki, T. Dietl, M. Buongiorno Nardelli, and J. Bernholc, Phys. Rev. Lett. 92, 037201 (2004).

[33] U. Fano, Phys. Rev. 124, 1866 (1961).

[34] A. Ernst, L. M. Sandratskii, M. Bouhassoune, J. Henk, and M. Lüders, Phys. Rev. Lett. 95, 237207 (2005).

[35] M. Dobrowolska, K. Tivakornsasithorn, X. Liu, J. K. Furdyna, M. Berciu, K. M. Yu, and W. Walukiewicz, Nat. Mater. 11, 444 (2012).

[36] P. W. Anderson, Phys. Rev. 124, 41 (1961).

[37] F. C. Zhang and T. M. Rice, Phys. Rev. B 37, 3759 (1988).

[38] W. Schairer and M. Schmidt, Phys. Rev. B 10, 2501 (1974).

[39] J. Schneider, U. Kaufmann, W. Wilkening, M. Baeumler, and F. Köhl, Phys. Rev. Lett. 59, 240 (1987).

[40] J. Okabayashi, A. Kimura, O. Rader, T. Mizokawa, A. Fujimori, T. Hayashi, and M. Tanaka, Phys. Rev. B 58, R4211 (1998).

[41] A. Fujimori, Basics of Strongly Correlated Electron Materials (in Japanese) (Uchida Rokakudo Publishing Co., Ltd., Tokyo, 2005).

[42] V. N. Strocov, R. Claessen, G. Nicolay, S. Hüfner, A. Kimura, A. Harasawa, S. Shin, A. Kakizaki, H. I. Starnberg, P. O. Nilsson, and P. Blaha, Phys. Rev. B 63, 205108 (2001). 